\title{
Design and Development of an Energy Efficient Algorithm for Data Aggregation in Wireless Sensor Network using Unsupervised Learning
}

\author{
Anitha C L, R Sumathi
}

\begin{abstract}
A wireless sensor network generally defined as the collection of sensors that are utilized to track and record the data in real-time on an ongoing basis from different applications. In comparison with other sensor nodes, data transmission obtained through sinks in WSN eliminates the energy in nearby nodes. This issue is identified as one of the major problems in a wireless sensor network. Two new algorithms were proposed in this research paper that mainly focused on the usage of machine learning algorithms to solve the data collection issue in the wireless sensor network. The algorithms proposed will able to create cluster heads to decrease energy usage, this will save about $50 \%$ of battery power consumption and mobile sinks are used to record the data from cluster heads in a network. Ultimately, current algorithms such as RLLO, DBRkM, CLIQUE, RL-CRC, and EPMS were compared.
\end{abstract}

Keywords-Agents, Cluster head, Markov decision process, Sink traversal, Reinforcement learning.

\section{INTRODUCTION}

To develop efficient algorithms for data aggregation in WSN, an important research field which is already discussed in the past few decades. Therefore, sleep and wake-up process is required to use sensor nodes to maximize their energy's network existence. This is how the theory of clustering works. As a result of the intermediate contact between base stations and rest of the Wireless sensor network, the nodes that lie near to base stations always get overloaded. It significantly reduces the efficiency of the network wireless sensor. Therefore, we have supported work in sink flexibility that has been found in WSNs to correctly handle the problem of hot spots and reduce the overhead of energy communication.

Mobile sink must be visited divisionally in each of its cluster headers to gather the information, which results in a longer mobile sink path that creates latency in data provision and increased energy usage. However, it is a demanding task to design a mobile sink travel path, because it depends heavily on network coverage, data provision, energy efficiency, and network life.

The machine learning techniques called reinforcement learning are applied and agents are trained in the new environments. A primary objective of agents is to create steps that will maximize future rewards. Such incentives later lead to an optimal strategy.

Revised Manuscript Received on December 12, 2019.

* Correspondence Author

Anitha C L*, Associate Professor, Dept of CSE, Kalpataru Institute of Technology, Tiptur-572201,Email:clanitha@gmail.com

Dr. R Sumathi, Professor \& Head Dept of CSE, SIT, Tumkur, India
Following the MDP (Markov Decision Process) framework, the components are states, indicators, and transformations between States. The usage of reinforcement learning approaches can therefore greatly improve the performance of WSNs. The following are the contribution to the research paper as follows:

1. A novel algorithm for creating a cluster head using a reinforcement learning algorithm (RLBCA).

2. The novel algorithm proposed to record and collects the data from the sensor nodes called ODMST.

\section{RELATED WORK}

This section discusses the literature survey of data collection in WSN. The authors [1] proposed the mobile sink geometric model, which worked extremely well on different performance metrics. TTDD was built in to differentiate the WSN into the wireless link node-based digital grids. The mobile sink path is based on the grid node which removes the hot spot problem. This grid-development process, however, consumes more $\mathrm{SN}$ energy.

In [2], the authors were focused here on energy-efficient PSO-based routing and clustering. Researchers have proposed a strategy that extends the network life by removing traffic from the gateways whose residual energy reaches a certain threshold cost, but the researchers also regarded the loss of the gateways as a function of the complete power depletion. The PSO algorithm used in the MPES algorithm [3] to increase network performance by using virtual clusters. The cluster heads are selected based on the mobility of mobile sinks. But the WSNs transmission coverage problem was not resolved by this algorithm. The authors in [4] focused on the issue of minimizing WSN delivery latency and using the random mobile sink in an aircraft, where the suggested algorithm is good at reducing data latency and route length. Nevertheless, the transmission issue impaired WSN quality. In [5], the authors proposed that an information distribution system, known as the tree overlay grid, be used to identify moving targets if several moving disks are located in WSN and require less power along with longer network life. The researchers [6] explained that local data can be shared for each node with adjacent nodes without unnecessary overhead output, allowing for an efficient rotation.

The researchers suggested DBRkM and RkM algorithms [7] create mobile sink paths. To determine a route via a hop interaction, the RkM algorithm joined SN's where the late-bound path created by DBRkM. Nevertheless, that 
sensor nodes have the same amount of data storage, so stay time for the phone sink is negligible. Researchers implemented the EAPC methodology in [8], which created a data collection course and selected qualifying sensors. The EAPC approach developed a minimal timbre at the base station. It increases network longevity and energy consumption, but a little less power is required and SN numbers are increasing. In [9], the author implemented I-UMDPC-based cluster routing where sensitive information is disrupted in time by the route. Nonetheless, this algorithm is more complex than current methods. The question of choosing an optimum cluster is formulated as an MDP showing good energy efficiency and reducing energy consumption when creating several intra and inter-cluster communications clusters [10].

Using enhancement learning, the CLIQUE algorithm [11] was used in data clusters that saved cluster head selection power so that nodes could determine whether or not to serve as packet-based cluster heads. A clustering algorithm to enhance learning is proposed in the research paper [12], that address the energy consumption and detection of the user in a wireless sensor network, which in turn slows down network coverage. The multi-target optimization survey, including several performance metrics and very useful algorithms. Researchers proactively proposed ways to improve network operation, coverage and reliability discovery with accurate results.

\section{III.PROBLEM DEFINITION}

Network Environment Model: The multiple sensor nodes are placed into an R-radius rectangle area. Figures 3.1 and 3.2 illustrate the basic architecture of the mobile sink. Every senor node is static and uniform [13]. There are similar sectors in the whole sensor network environment. Source nodes are free to adjust transmission power to target nodes according to the distance.

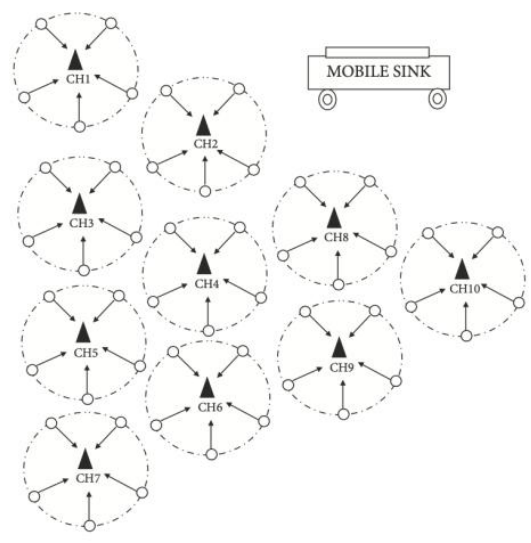

Figure 3.1: Mobile Sink Architecture

Basic Assumptions. In this research study, we have taken as follows:

1. The static and homogeneous existence of all deployed WSN nodes.

2. The initial energy of all WSN nodes is the same [14].

3. In the network environment, no physical barriers/obstacles are found.

4. The handheld sink [15] is capable of gathering information at the right time from the cluster heads.
Energy Model: The first radio-energy model [14] was considered as an energy method for measuring energy usage. The equations shown below shows the energy usage can be measured in the following ways: absorption and transmission.

$$
\begin{gathered}
\left.E_{T x}(l, d)\right)= \begin{cases}l \cdot E_{\text {elec }}+l \cdot \varepsilon_{f s} \cdot \mathrm{d}^{2}, & \text { when } \mathrm{d}_{0}>d \\
l \cdot E_{\text {elec }}+l \cdot \varepsilon_{m p} \cdot \mathrm{d}^{4}, & \text { when } \mathrm{d}_{0} \leq \mathrm{d}\end{cases} \\
E_{R x}(l)=l \cdot E_{\text {elec }}
\end{gathered}
$$

Problem Formulation: Network life and energy consumption are the key performance variables for WSN. When a first node dies, the lifetime of WSN is counted. Figure 3.2 represents the proposed architecture of the mobile sink traversal path. Figure 3.2 indicates that Mobile sink immediately declares its current position for all cluster heads. If necessary, cluster heads must give their message to the Mobile sink. The distance between cluster heads and Mobile sink is then measured and the path is then established for data collection. If cluster heads are submitting their query once more during the traversal process of sensor nodes, then the Mobile Sink updates the path according to the shortest distance and executes this for data collection.

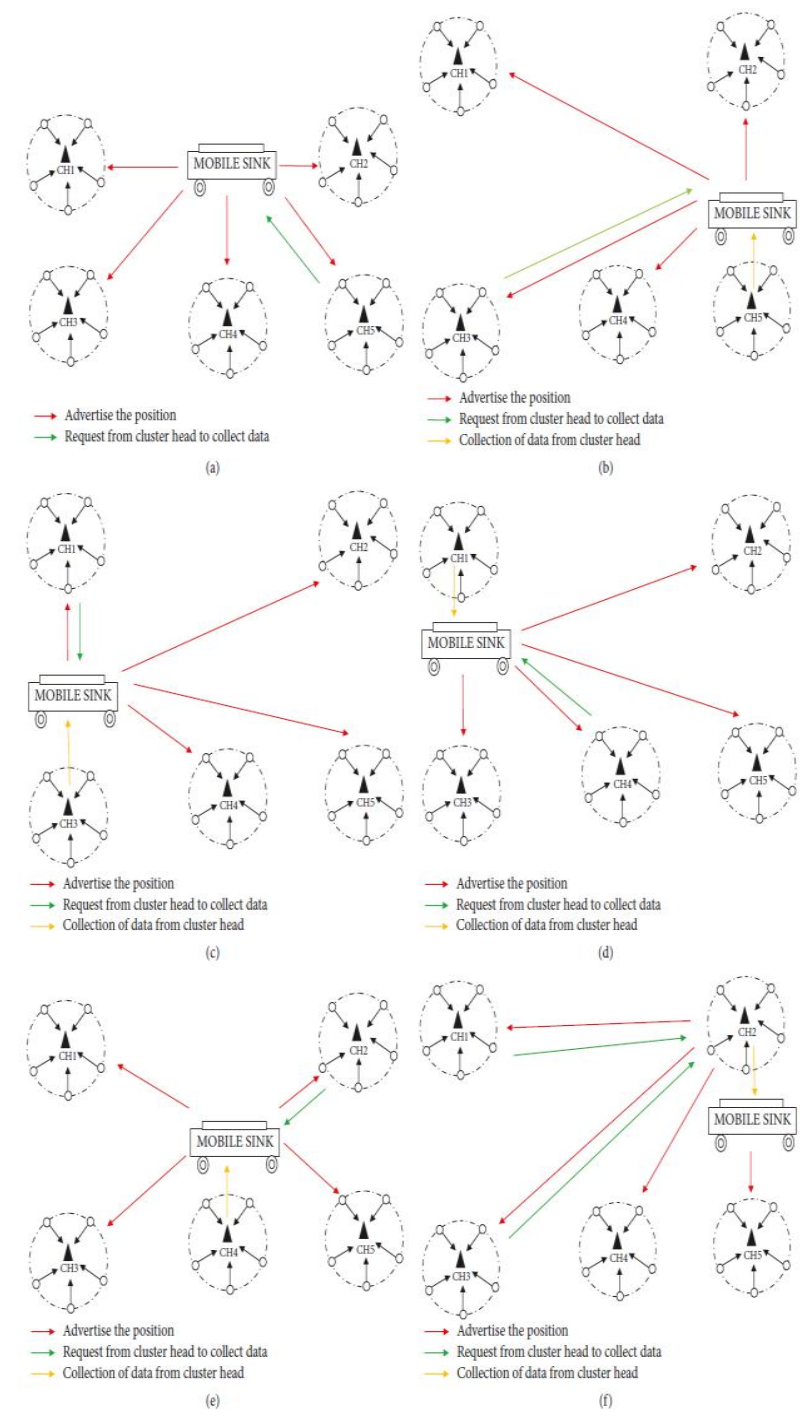

Figure 3.2: Architecture of Mobile Sink traversal path 
Reinforcement Learning (RL)

RL has several basic components such as reward, agent, state, policy, action, model and value function of the environment. Figure 3.3 presents the RL learning process.

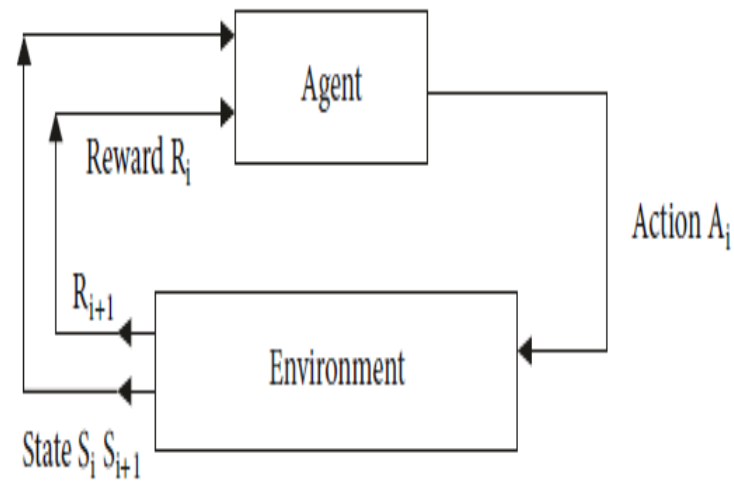

Figure 3.3: RL learning process

\section{PROPOSED ALGORITHMS}

Clustering of SNs by Using Reinforcement Learning: We have suggested in this chapter RLBCA where the WSN node acts as a learning agent to build up clusters based on certain policies in the energy level of their closest neighbors. Cluster search is calculated for the Markov decision process (MDP) [15]. The MDP includes government, action, rewards, and policies. In order to learn from networking, the learning agent uses a time-difference process. For Clustering the RL template is used (state, Action, and Reward). Markov decision process (MDP) is a 4-tuple [ set of states (S), Transitional function (T), Action(A), Reward (R)]. The agent chooses an action for all states as shown in Figure 4.1. The selected action subsequently calculates the cluster energy consumption. The rewards resulting from energy consumption determined to take the right action. The decision that was made raises the current state to the next state and the current action to the next action. The best policies are determined by the agents, maximizing the gain. The data obtained from various nodes are collected and generalized without any noise, this can be achieved only when we train the network in such a way that network should adapt to the environment.

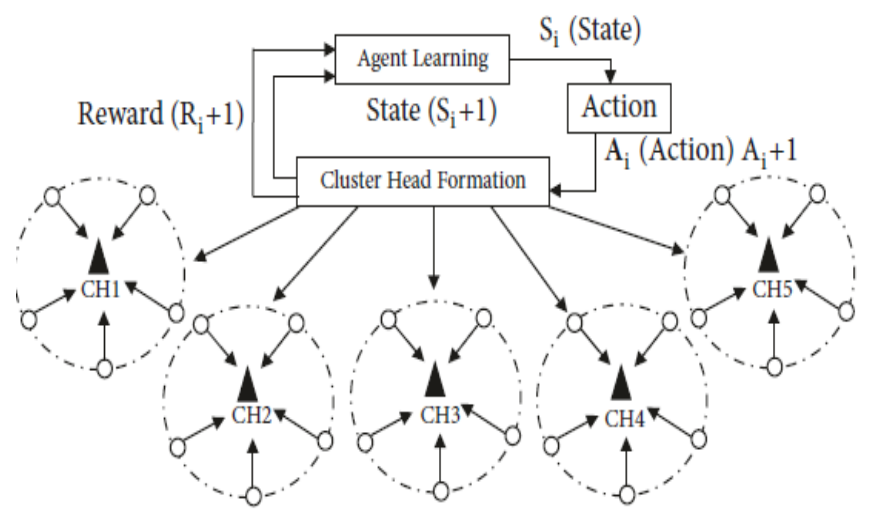

Figure 4.1: Reinforcement learning for creation of cluster heads
The algorithm is as follows:

Step L. Initially all sensor nodes sends hello message packet to show their residual energy and current position.

Step 2. The learning agent records the total number of neighbour nodes and their residual energy. Periodically the residual energy of each sensor nodes is set and return value of the node is set to zero.

Step 3. Based upon step 2, duster head formation probability is computed. The base station selects the optimal number of cluster heads among the desired cluster heads and creates the list.

Step 4. The base station announces the list of eligble cluster heeds.

Step 5. The newly formed duster heads send advertisement packets to their nearest

Neighbours for communication purpose.

Step 6. The state-action Q.-values [10] are updated by reward function (equation $(*))$ and $Q$-matrix (equation $(* *))$ to achieve the optimal policy (equation $(* * *))$;

Reward calculation

$\left.r_{i+1}^{t} \vdash \operatorname{Avg}(r) d_{E_{i}+1}^{t}, r w d_{d, i+1}^{t}\right)$

Q-matrix updation

$\left.Q_{i+1}^{t} \leftarrow Q_{i+1}^{t}+\alpha r_{i+1}^{t}+y \max Q_{1}\left(Q_{i+1}^{t}-Q_{i}^{t}\right)\right] \quad(* *)$

Optimal policy

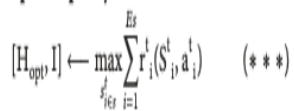

Step7, if the current nodes sesidual energy is greater than other neighbour's nodes, the sensor node with higher residual energy is elected as a cluster head for next subseguent round.

Step 8. Repeat step 1 to step?.

\section{Mobile Sink Traversal Algorithm: ODMST}

The RLBCA composed cluster heads; the ODMST algorithm collects data from the cluster heads obtained from the above algorithm.

Step1: Cluster heads are obtained from the Above algorithm.

Step2: Randomly placed mobile sink nodes Broadcast the position to cluster heads.

Step3: The cluster head send the request to Mobile sink node to visit the message Packet.

Step4: Mobile sink stores the message in Routing table to identify the distance.

Step5: If the mobile sink receives the multiply Message:

Than shortest traversal path is Created and calculates the distance.

Step6: Jump to step 3.

\section{PERFORMANCE EVALUATIONS}

We have contrasted the proposed algorithm with existing algorithms, including the RLLO, DBRkM, CLIQUE, RL-CRC, and EPMS. In the area of 400x 400-meter square, the network setting includes 1000 sensor nodes and having $0.5 \mathrm{~J}$ initial energy consumption. We have used OMNeT++ simulator based on the following simulation parameters:

- $\quad$ Range communication $=100 \mathrm{~m}$.

- $\quad$ Packet size $=2000$ bits .

- $\quad$ Learning parameter $=0.5,0.7,0.9$ and 0.11 .

- Speed of sensor node $=4,5,6,7,8$, and $9 \mathrm{~m} / \mathrm{s}$.

- Random deployment. 


\section{RESULT ANALYSIS}

The findings were calculated by the following performance metrics, compared with other algorithms such as RLLO, DBRkM, CLIQUE, RL-CRC, and EPMS:

1. Loss of Routing energy: Each cluster head of the WSN absorbs energy. Cluster heads not part of the sink route are said to be idle to save energy.

2. Lifetime network: indicates the duration of nodes.

3. Learning time: indicates the performance of the WSN, operation increases as the learning rate goes up and the energy consumption decreases.

4. Algorithm convergence: This is the algorithm output expressed in two ways: speed and convergence order.

5. Description of Square Error (SSE): It provides a generic cluster method of analysis. The SSE demonstrates cluster heads efficiency according to power management.

After detailed simulation in OMNeT++, the Reinforcement cluster algorithm is establishing the solution directly from the action pair. It depends mainly on the speed of learning, the discount variable and the method of taking an intervention. We modelled proposed method to check our algorithm's convergence over 4000 episodes and tested its efficiency as shown in Figure 5.1. The results revealed that the Reinforcement cluster algorithm produces the optimal solution because of its quicker network convergence and shorter learning time where we have used Q-learning for clustering.
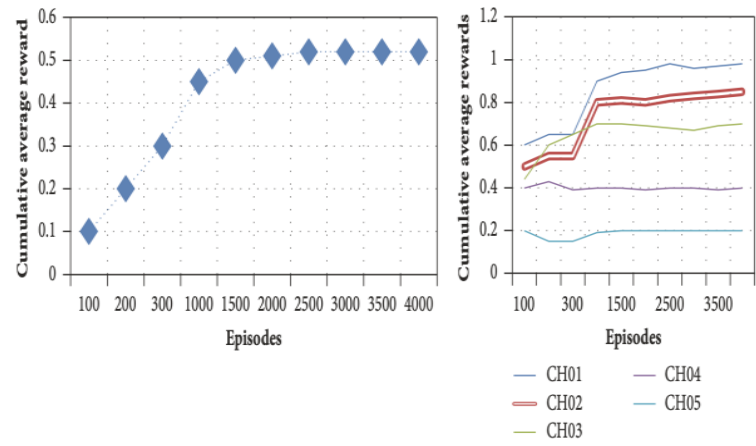

(a) Expected cumulative rewards and algorithms convergence
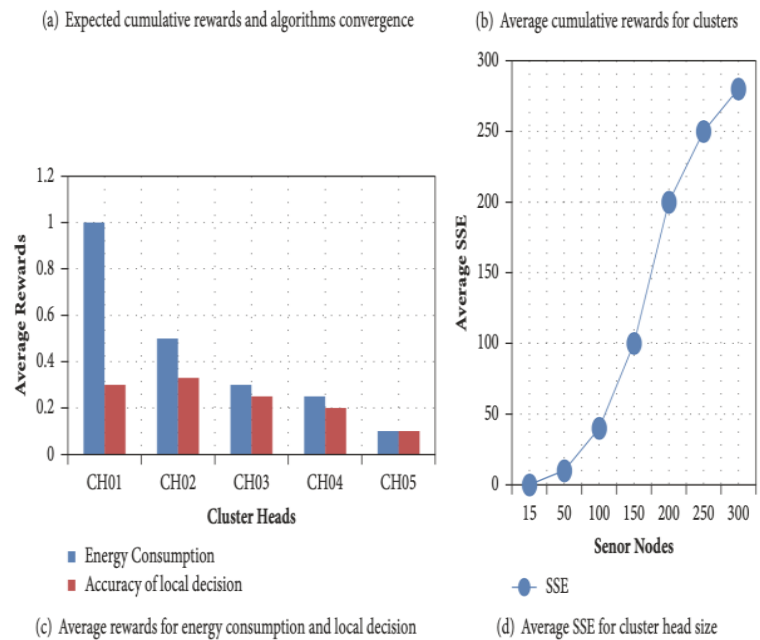

Figure 5.1: Result analysis of RLBCA
Figure 5.2 shows as the number of iteration increases, the sink traversal algorithm's energy consumption is comparatively lower than other algorithms RLLO, DBRkM, CLIQUE, RL-CRC, and EPMS.
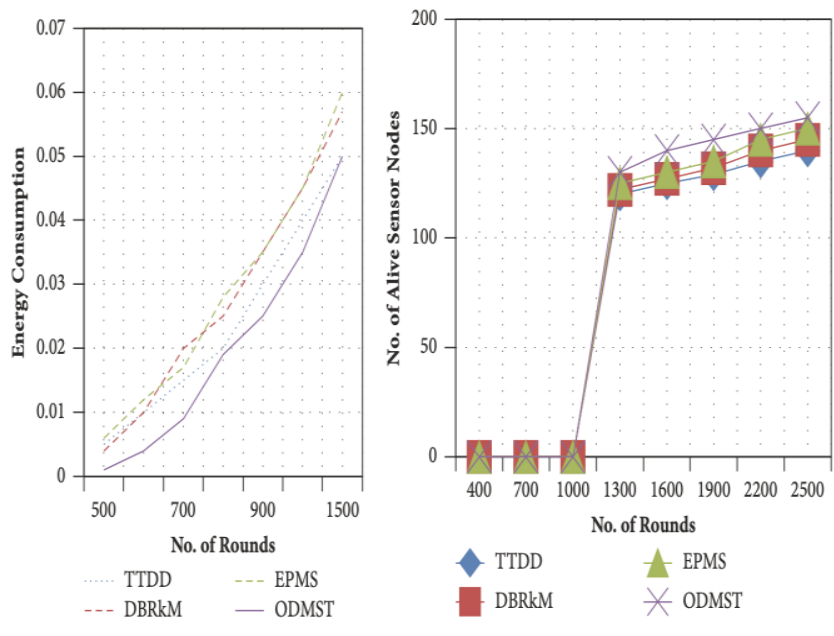

(b) Network lifetime

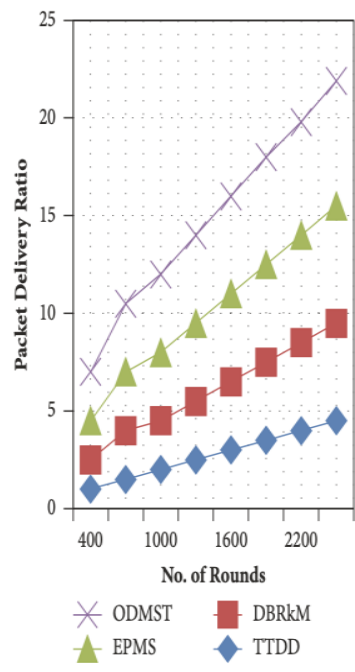

(c) PDR ratio

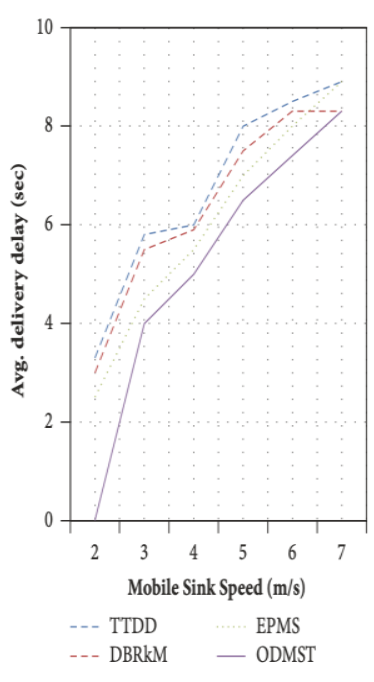

(d) Average end to end delay

\section{Figure 5.2: Result analysis of ODMST}

\section{CONCLUSIONS}

The two novel learning algorithms are proposed mainly to solve energy consumption in WSN using reinforcement learning. The cluster heads are used to record the data depending on their demand, which primarily saves energy consumption from the cellular sink and boosts network life. Simulation results showed and compared with different algorithms, the proposed algorithms accurately generated cluster head and collect data from cluster heads via mobile sinks. This research study motivates us to test on a large scale the scalability and integration in WSN.

\section{REFERENCES}

1. G. Chen, J.-S. Cheuh, M.-T. Sun, T.-C. Chiang, and A. A.-K. Jeng, "Energy-efficient mobile targets detection in the presence of mobile sinks," Computer Communications, vol. 78, pp. 97-114, 2018. 
2. N. Ghosh and I. Banerjee, "An energy-efficient path determi- nation strategy for mobile data collectors in wireless sensor network," Computers and Electrical Engineering, vol. 48, pp. 417-435, 2018.

3. T. T. T. Le and S. Moh, "Reinforcement-Learning-Based Topol- ogy Control for Wireless Sensor Networks," in Proceedings of the Grid and Distributed Computing 2018, pp. 22-27.

4. M. Zareei, A. K. M. M. Islam, C. Vargas-Rosales, N. Mansoor, S. Goudarzi, and M. H. Rehmani, "Mobility-aware medium access control protocols for wireless sensor networks: A survey," Journal of Network and Computer Applications, vol. 104, pp. 21-37, 2018.

5. J. Randlov and P. Alstrom, "Learning to drive a bicycle using reinforcement learning and shaping," in Proceeding of the fifteenth international conference on machine learning, ACM DL, pp. 463-471, 2018.

6. H.Ahmadi,F.Viani,andR.Bouallegue,"An accurate prediction method for moving target localization and tracking in wireless sensor networks," Ad Hoc Networks, vol. 70, pp. 14-22, 2018.

7. A. Forster and A. L. Murphy, "CLIQUE: Role-free clustering with Q-learning for wireless sensor networks," in Proceed- ings of the 2009 29th IEEE International Conference on Distributed Computing Systems Workshops, ICDCS, 09, pp. 441-449, Canada, June 2018.

8. P. Montague, "Reinforcement Learning: An Introduction, by Sutton, R.S. and Barto, A.G.," Trends in Cognitive Sciences, vol. 3, no. 9, p. 360, 2017.

9. M. Elshrkawey, S. M. Elsherif, and M. Elsayed Wahed, "An Enhancement Approach for Reducing the Energy Consump- tion in Wireless Sensor Networks," Journal of King Saud University Computer and Information Sciences, vol. 30, no. 2, pp. 259-267, 2017.

10. G. Dangelo, D. Diodati, A. Navarra, and C. M. Pinotti, "The Minimum $\square$-Storage Problem: Complexity, Approximation, and Experimental Analysis," IEEE Transactions on Mobile Com- puting, vol. 15, no. 7, pp. 1797-1811, 2017.

11. I. Mustapha, B. M. Ali, M. F. A. Rasid, A. Sali, and H. Mohamad, "An energy-efficient spectrum-aware reinforcement learning- based clustering algorithm for cognitive radio sensor networks," Sensors, vol. 15, no. 8, pp. 19783-19818, 2017.

12. W. Wen, S. Zhao, C. Shang, and C.-Y. Chang, "EAPC: EnergyAware Path Construction for Data Collection using Mobile Sink in Wireless Sensor Networks,” IEEE Sensors Journal, 2017.

13. Y. Gu, F. Ren, Y. Ji, and J. Li, "The evolution of sink mobility management in wireless sensor networks: a survey," IEEE Communications Surveys \& Tutorials, vol. 18, no. 1, pp. 507-524, 2016.

14. S. Guo, X. Wang, and Y. Yang, "Topology control for maximizing network lifetime in wireless sensor

15. networks with mobile sink," in Proceedings of the 10th IEEE International Conference on Mobile Ad-Hoc and Sensor Systems, MASS 2013, pp. 240-248, China, October 2016.

16. W. Osamy and A. M. khedr, "An algorithm for enhancing coverage and network lifetime in Cluster Based wireless sensor networks,' International Journal of Communication Networks and Information Security, vol. 10, pp. 1-9, 2016.M. Wiering and M. van Otterlo, "Adaptation learning and optimization," Reinforcement Learning state of the art, Book, vol. 12, pp. 3-326, 2016, ISSN 1867-4534.

17. Manjeswar, A.; Agrawal, D.P. TEEN: "A protocol for enhanced efficiency in wireless sensor networks". In Proceedings of $1 \mathrm{st}$ International Workshop on Parallel and Distributed Computing Issues in Wireless Networks and Mobile Computing, San Francisco, CA, USA, 2016; p. 189.

18. Olivier Dousse, PetteriMannersalo, Patrick Thiran "Latency of Wireless Sensor Networks with Uncoordinated Power Saving Mechanisms" MobiHoc'04, May 24-26, 2016, Roppongi, Japan.

19. Martorosyan, A.; Boukerche, A.; NelemPazzi, R.W. Taxonomy of cluster-based routing protocols for wireless sensor networks". In International Symposium on Parallel Architectures, Algorithms, and Networks, Sydney, NSW,Australia, May 7-9, 2016; pp. 247-253.

20. M. C.Vuran and I. F. Akyildiz, "Spatial Correlation-Based Collaborative Medium Access Control in Wireless Sensor Networks," in IEEE/ACM Transactions on Networking, vol. 14, no. 2, pp. 316 -329 , April 2016.

\section{AUTHORS PROFILE}

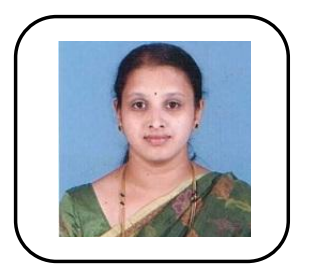

Mrs. Anitha C L, Associate Professor in the Dept of CSE, KIT, Tiptur, India. She is currently pursuing $\mathrm{Ph} . \mathrm{D}$ under VTU, Belagavi. She has published several research papers, international conference papers since 2011 She is an active member of ISTE.

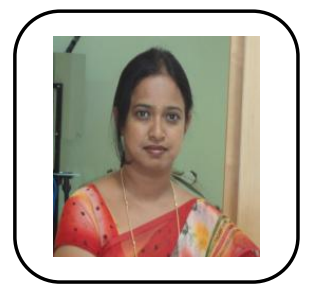

Dr. R Sumathi, Professor \& Head Dept of CSE, SIT, Tumkur, India. She has published several research papers in international conferences and journals. She had 22 years of academic experience. Her area of interest is routing in wireless sensor networks, Adhoc networks and distributed computing. 